\title{
Does bilateral uterine artery ligation have negative effects on ovarian reserve markers and ovarian artery blood flow in women with postpartum hemorrhage?
}

\author{
Fatma Ferda Verit', Orkun Çetin², Seda Keskin³, Hürkan Akyol', Ali Galip Zebitay' \\ 'Department of Obstetrics and Gynecology, Suleymaniye Maternity, Research and Training Hospital, University of Health Sciences, Istanbul; \\ ${ }^{2}$ Department of Obstetrics and Gynecology, Yuzuncu Yil University Medical Faculty, Van; ${ }^{3}$ Department of Obstetrics and Gynecology, Ordu University \\ Medical Faculty, Ordu, Turkey
}

Objective: Bilateral uterine artery ligation (UAL) is a fertility-preserving procedure used in women experiencing postpartum hemorrhage (PPH). However, the long-term effects of this procedure on ovarian function remain unclear. The aim of this study was to investigate whether bilateral UAL compromised ovarian reserve and ovarian blood supply.

Methods: This prospective study included 49 women aged between 21 and 36 years who had undergone a cesarean section for obstetric indications. Of these, 25 underwent uterine bilateral UAL to control intractable atonic PPH. The control group consisted of 24 women who had not undergone bilateral UAL. Standard clinical parameters, the results of color Doppler screening, and ovarian reserve markers were assessed in all participants at 6 months after surgery. The clinical parameters included age, parity, cycle history, body mass index, and previous medication and/or surgery. Color Doppler screening findings included the pulsatility index (PI) and resistance index (RI) for both the uterine and ovarian arteries. The ovarian reserve markers included day 3 follicle-stimulating hormone (FSH) levels, antral follicle count, and anti-Müllerian hormone (AMH) levels.

Results: There were no significant differences in the ovarian reserve markers of day $3 \mathrm{FSH}$ levels, antral follicle count, and AMH levels between the study and control groups ( $p>0.05$ for all). In addition, no significant differences were observed in the PI and Rl indices of the uterine and ovarian arteries ( $p>0.05$ for all).

Conclusion: In this study, we showed that bilateral UAL had no negative effects on ovarian reserve or ovarian blood supply, so this treatment should be used as a fertility preservation technique to avoid hysterectomy in patients experiencing PPH.

Keywords: Ovarian blood flow; Ovarian reserve; Uterine artery ligation; Uterine blood flow

\section{Introduction}

Postpartum hemorrhage (PPH) is a life-threatening complication.

Received: Sep 5, 2018 · Revised: Nov 10, 2018 · Accepted: Dec 3, 2018 Corresponding author: Fatma Ferda Verit

Department of Obstetrics and Gynecology, Suleymaniye Maternity, Research and Training Hospital, University of Health Sciences, Istanbul, Turkey Tel:+90-212-6645355 Fax:+90-212-4169814 E-mail:fverit@gmail.com

This is an Open Access article distributed under the terms of the Creative Commons Attribution Non-Commercial License (http://creativecommons.org/licenses/by-nc/4.0/) which permits unrestricted non-commercial use, distribution, and reproduction in any medium, provided the original work is properly cited.
Uterine atony is considered to be the most common cause [1]. The other known risk factors for PPH include placenta accreta, placental abruption, retained placenta, genital tract lacerations, uterine rupture, and coagulation disorders [1].

Bimanual uterine compression, pharmacological therapies, and intrauterine balloon tamponade are the first-line therapies for atonic PPH [2]. If these therapies fail, surgery may be needed to control the hemorrhage. Hysterectomy is the definitive surgical procedure for blood loss; however, it is a radical procedure, especially in young women [2]. For this reason, several fertility-preserving surgical tech- 
niques have been developed, such as uterine artery ligation (UAL), internal iliac artery ligation, the B-Lynch technique, and modified compression sutures [2].

UAL is one of the most popular fertility-preserving surgical techniques. It is easy to perform and effective for controlling PPH [1]. It is also relatively safe and allows patients to bear more children in the future. Furthermore, is success rate is over $90 \%$ [3].

Diminished ovarian reserve is a major cause of infertility. Widelyused markers of ovarian reserve, which is a proxy for the likelihood to conceive, include age, follicle-stimulating hormone (FSH) levels, antral follicle count (AFC), anti-Müllerian hormone (AMH) levels, and inhibin B levels.

It is widely known that the blood supply to the ovaries comes from the ovarian arteries which are direct branches of the abdominal aorta, and the uterine arteries. Many studies have reported that hysterectomy can lead to decreased ovarian reserve and early menopause, even when the ovaries are not removed [4-7]. FSH levels were found to be elevated in women who had undergone hysterectomies, even when one ovary had been removed [5]. Another study reported that inhibin B levels had decreased at 6 and 12 months after abdominal hysterectomy [6]. Finally, reduced ovarian reserve was observed after uterine artery embolization (UAE) [4]. However, the effects of UAL on ovarian reserve and ovarian artery blood flow have not been adequately studied. The aim of this study was to investigate whether UAL had any negative effects on ovarian reserve markers and ovarian blood flow in young women with PPH.

\section{Methods}

This prospective study included 49 women aged between 21 and 36 years who received care at the Department of Obstetrics and Gynecology at Suleymaniye Maternity, Research and Training Hospital between October 2012 and October 2013. All participants who underwent a cesarean section for obstetric indications were included in the study, unless they satisfied the exclusion criteria described below. Of the 49 participants, 25 received uterine bilateral UAL by the same surgeon to control intractable atonic PPH. The control group consisted of 24 healthy women who had not undergone bilateral UAL or any other interventions for PPH. Fundal massage, bimanual compression, oxytocin administration (40 IU in $500 \mathrm{~mL}$ intravenously), methylergonovine maleate (0.20-0.40 mg, intramuscularly), and rectal misoprostol (0.8-1 mg rectally) were applied to all patients before bilateral UAL.

Patients with other causes of PPH, such as traumatic PPH, disseminated intravascular coagulation, bleeding disorders, retained placenta, and uterine rupture were excluded from the study. Other exclusion criteria were receiving B-Lynch or other hemostatic sutures, stepwise uterine devascularization, internal iliac artery ligation, or hysterectomy; being older than 37 years; and obesity (body mass in$\operatorname{dex}[\mathrm{BMI}],>30 \mathrm{~kg} / \mathrm{m}^{2}$ ). None of the patients had previously been diagnosed with endometriosis or had any clinical symptoms thereof, and they had no other known risk factors for diminished ovarian reserve, such as infertility, a history of ovarian surgery, the presence of neoplastic disease, infectious diseases, autoimmune diseases, liver or kidney disease, diabetes, vascular disease, irregular menstrual cycles, smoking, or alcohol consumption.

Blood transfusion, plasma expanders, and fibrinogen were administered depending on the patients' needs. The study was approved by our Institutional Review Board (IRB No. 13.06.2012-03). Written informed consent was obtained from all patients.

PPH was defined as blood loss greater than 1,000 mL following a cesarean section. Intractable PPH was defined as PPH that persisted despite standard medical treatment (oxytocin, methylergonovine maleate, and misoprostol), fundal massage, and bimanual compression.

Bilateral UAL was performed $2 \mathrm{~cm}$ below the Kerr incision (lower segment transverse). A 0-Vicryl absorbable suture (Ethicon, Neuilly-surSeine, France) was placed from the anterior to posterior aspects of the myometrium 2-3 cm medial to the descending part of the uterine vessels through an avascular space in the broad ligament and tied. Uterine tonus and bleeding were then controlled after the procedure.

Standardized clinical parameters, color Doppler screening findings, and ovarian reserve markers were assessed in all participants at 6 months after surgery. The clinical parameters included age, parity, cycle history, BMI, and previous medication and/or surgery. Color Doppler screening findings included the pulsatility index (PI) and resistance index (RI) for both the uterine and ovarian arteries. The ovarian reserve markers included day $3 \mathrm{FSH}$ levels, $\mathrm{AFC}$, and $\mathrm{AMH}$ levels.

\section{Blood sample collection}

Blood samples were collected at 9:00 and 11:00 AM after an overnight fast between days 3 and 5 of the menstrual cycle. The blood samples were then centrifuged within 2 hours after being obtained and assessed on the same day. AMH concentrations were measured with an enzymatically amplified two-sided immunoassay (AMH Gen II Elisa A79765; Beckman Coulter, Ireland).

\section{Doppler scanning}

Ultrasound examinations were performed using a Voluson 730 Expert (GE Healthcare, Buckinghamshire, UK) apparatus with a 4-9 MHz endovaginal probe between days 20 and 22 of the menstrual cycle. The ultrasound settings were standardized, with a pulse repetition frequency of $1.3 \mathrm{kHz}$ for the uterine and ovarian arteries and $1.1 \mathrm{kHz}$ for the spiral artery to evaluate a minimum flow velocity of $5 \mathrm{~cm} / \mathrm{sec}$, gain of 3.4, a low wall motion filter (1), the harmonic setting at low, and power at $100 \%$. The same procedure was applied to all patients. 
Transverse and sagittal imaging of the uterus and ovaries was performed using an endovaginal probe. The spiral arteries were assessed within a 1-mm area parallel to the myometrium-endometrium border by color Doppler. The descending branches of the uterine artery were measured at the level of the internal os of the cervix uteri. The ovarian arteries were evaluated as the large vessels at the level of the ovarian hilum. The PI and RI of both the right and left uterine and ovarian arteries were calculated. Average values were recorded.

\section{Statistical analysis}

The baseline characteristics of the groups were shown as the mean and standard deviation. The laboratory and anthropometric parameters of patients were compared using the Student $t$-test for parametric parameters and the Mann-Whitney test for nonparametric parameters. Differences were considered significant at $p<0.05$.

\section{Results}

The demographic characteristics and ovarian reserve markers of the patients are summarized in Table 1. There were no differences in terms of age, BMI, parity, day 3 FSH levels, AFC, and AMH levels between the groups ( $p>0.05$ for all) (Table 1). The results for uterine and ovarian artery $\mathrm{PI}$ and RI are shown in Table 2. Likewise, no significant differences were observed in uterine and ovarian artery PI and
RI among patients who underwent bilateral UAL and those who did not ( $p>0.05$ for all) (Table 2 ).

\section{Discussion}

The uterine arteries are sometimes disrupted during surgical procedures such as UAL, hysterectomy, and UAE, resulting in a lack of ovarian blood supply from the uterine arteries. The unresolved question is whether these procedures harm the patient's ovarian reserve. This issue is important because fertility-preserving procedures are often performed in young patients, and we must inform patients and/or their families about not only the reproductive potential of the uterus, but also their ovarian reserve. However, data about the ovarian reserve of these patients are limited. To the best of our knowledge, this is the first prospective study to find that bilateral UAL did not have any negative effects on ovarian reserve markers such as day $3 \mathrm{FSH}$ levels, AFC, and AMH levels. Furthermore, uterine and ovarian blood flow was not found to have been compromised in patients who underwent UAL.

Many studies have investigated the effects of hysterectomy and UAL on ovarian reserve [5,6,8-11]. It has been claimed that hysterectomy compromises ovarian reserve and may accelerate menopause [8]. However, this suggestion was based on the vasomotor symptoms of menopause, which are not a reliable predictor of ovarian re-

Table 1. Demographic characteristics and ovarian reserve markers of the study and control groups

\begin{tabular}{|c|c|c|c|}
\hline Variable & Bilateral uterine artery ligation group $(n=25)$ & Control group $(n=24)$ & $p$-value \\
\hline Age (yr) & $28.16 \pm 2.74$ & $27.42 \pm 2.58$ & 0.33 \\
\hline Body mass index $\left(\mathrm{kg} / \mathrm{m}^{2}\right)$ & $25.81 \pm 1.63$ & $25.65 \pm 1.67$ & 0.74 \\
\hline Parity & $1.80 \pm 0.70$ & $1.71 \pm 0.75$ & 0.66 \\
\hline Day 3 FSH (mlU/mL) & $7.90 \pm 1.11$ & $7.52 \pm 1.11$ & 0.24 \\
\hline Antral follicle count & $10.32 \pm 1.99$ & $11.25 \pm 2.04$ & 0.11 \\
\hline $\mathrm{AMH}(\mathrm{ng} / \mathrm{mL})$ & $2.17 \pm 0.50$ & $2.48 \pm 0.64$ & 0.07 \\
\hline
\end{tabular}

Values are presented as mean \pm standard deviation.

$\mathrm{FSH}$, follicle-stimulating hormone; $\mathrm{AMH}$, anti-Müllerian hormone.

Table 2. Color Doppler parameters of the study and control groups

\begin{tabular}{lccc}
\hline Variable & Bilateral uterine artery ligation group $(n=25)$ & Control group $(n=24)$ & $p$-value \\
\hline Right uterine artery RI & $0.79 \pm 0.07$ & $0.77 \pm 0.06$ & 0.61 \\
Left uterine artery RI & $0.74 \pm 0.07$ & $0.75 \pm 0.06$ & 0.76 \\
Right uterine artery PI & $1.78 \pm 1.13$ & $1.75 \pm 1.10$ & 0.33 \\
Left uterine artery PI & $1.75 \pm 1.19$ & $1.73 \pm 1.10$ & 0.78 \\
Right ovarian artery RI & $0.64 \pm 0.06$ & $0.64 \pm 0.03$ & 0.56 \\
Left ovarian artery RI & $0.70 \pm 0.07$ & $0.70 \pm 0.05$ & 0.83 \\
Right ovarian artery PI & $1.23 \pm 0.23$ & $1.26 \pm 0.13$ & 0.56 \\
Left ovarian artery PI & $1.24 \pm 0.13$ & $1.23 \pm 0.12$ & 0.87 \\
\hline
\end{tabular}

Values are presented as mean \pm standard deviation.

$\mathrm{RI}$, resistance index; PI, pulsatility index. 
serve. Ovarian function has also been assessed by more reliable ovarian reserve markers in other studies. Cooper and Thorp [5] reported that FSH levels were slightly, but nonsignificantly, elevated in women who underwent hysterectomy without oophorectomy. Another study demonstrated that abdominal hysterectomy without oophorectomy did not affect ovarian reserve and that $\mathrm{FSH}$, luteinizing hormone (LH), and estradiol were at premenopausal levels at 6 months after surgery [9]. Another study reported that FSH and LH were unchanged at 6 and 12 months post-hysterectomy, but inhibin B levels had decreased [6]. However, only $12.9 \%$ of those women showed findings compatible with ovarian failure [6]. Furthermore, inhibin B is no longer accepted as a reliable marker of ovarian reserve $[12,13]$. Lee et al. [10] also found that hysterectomy did not change AMH levels or ovarian arterial blood flow, defined in terms of PI and RI values.

Some studies have also investigated the effects of radical hysterectomy $(\mathrm{RH})$ on ovarian reserve [14-16]. It has been suggested that $\mathrm{RH}$ with ovarian preservation did not alter the age of menopause [16]. Ellsworth et al. [14] found that $\mathrm{RH}$ conserved ovarian function in $80 \%$ of patients. Another study reported that abdominal radical trachelectomy did not affect ovarian reserve or response to ovarian stimulation [15].

Early studies claimed that UAE might lead to ovarian failure via utero-ovarian anastomoses. Inadvertent embolization of the ovarian circulation has been suggested to be the mechanism of post-UAE ovarian insufficiency $[17,18]$. The size and the type of embolic agent, extent of embolization, and the end point of embolization have also been proposed as factors associated with ovarian failure [19]. However, ovarian artery embolization has not been clearly demonstrated to affect ovarian reserve, and multiple studies have indicated that it does not seem to increase the risks of permanent amenorrhea, premature menopause, or menopausal symptoms compared with UAE [19-21]. Moreover, the natural flow of the embolic agent is not towards the ovaries, and flow to the ovaries may not be present under normal conditions during embolization.

Recent studies demonstrated that UAE had no effect on ovarian reserve markers in patients younger than 40-45 years of age [19,22,23]. No significant differences in $\mathrm{AMH}$ and FSH levels were found in women younger than 45 years at 12 months after UAE [22]. Furthermore, no cases of amenorrhea were observed in that group [22]. It was also reported that UAE did not affect ovarian reserve in women younger than 40 years and that ovarian reserve tests did not show any statistically significant differences in those subjects; those findings are similar to our results $[23,24]$. Another study showed that $48 \%$ of UAE patients under 40 years old who desired fertility achieved term pregnancy [25].

In this study, we found that the uterine artery blood supply was not compromised after bilateral UAL. The result is especially important for young women who are expecting future pregnancies, because it is widely known that uterine blood flow is essential for promoting a receptive endometrium, embryo implantation, trophoblast invasion, and successful pregnancy [26]. Chang et al. [27] reported that bilateral UAL through laparoscopic myomectomy did not decrease uterine artery $\mathrm{PI}$ and $\mathrm{RI}$ values at 3 months after surgery. Moreover, other studies have also demonstrated that uterine artery PI and RI values did not change after bilateral internal iliac artery ligation [28,29].

Preserving ovarian blood supply during surgical procedures is important, especially in young women of reproductive age. Bilateral UAL is one of the most important fertility-preserving techniques in patients experiencing $\mathrm{PPH}$, and our study showed that it did not compromise ovarian blood supply and ovarian reserve in those patients, suggesting that UAL should be used to avoid hysterectomy in cases of PPH.

There may be some explanations for why uterine blood flow was restored 6 months after surgery. We used absorbable suture material, and the recanalization rate of the uterine artery was found to be high at 6 months after surgery [30]. Studies have also documented the possibility of increased collateral blood flow after bilateral UAL [31]. The patients were re-evaluated at 6 months after surgery in this study because Vicryl theoretically undergoes complete absorption within 6 months.

However, there were some limitations of this study. First, the study size was relatively small. Second, we were not able to evaluate the ovarian reserve markers of the patients before pregnancy and surgery, because it was impossible to determine which patients would become pregnant, undergo cesarean sections, develop PPH, and undergo bilateral UAL.

In conclusion, bilateral UAL did not have any negative effects on ovarian reserve. Furthermore, this surgery did not disrupt uterine and ovarian blood flow. More studies with larger groups of patients are needed to support this conclusion.

\section{Conflict of interest}

No potential conflict of interest relevant to this article was reported.

\section{References}

1. Sebghati M, Chandraharan E. An update on the risk factors for and management of obstetric haemorrhage. Womens Health (Lond) 2017;13:34-40.

2. Doumouchtsis SK, Nikolopoulos K, Talaulikar V, Krishna A, Arulkumaran S. Menstrual and fertility outcomes following the surgical management of postpartum haemorrhage: a systematic review. BJOG 2014;121:382-8. 
3. Joshi VM, Otiv SR, Majumder R, Nikam YA, Shrivastava M. Internal iliac artery ligation for arresting postpartum haemorrhage. BJOG 2007; 114:356-61.

4. Hehenkamp WJ, Volkers NA, Broekmans FJ, de Jong FH, Themmen AP, Birnie $E$, et al. Loss of ovarian reserve after uterine artery embolization: a randomized comparison with hysterectomy. Hum Reprod 2007;22:1996-2005.

5. Cooper GS, Thorp JM Jr. FSH levels in relation to hysterectomy and to unilateral oophorectomy. Obstet Gynecol 1999;94:96972.

6. Nahas E, Pontes A, Traiman P, NahasNeto J, Dalben I, De Luca L. Inhibin $B$ and ovarian function after total abdominal hysterectomy in women of reproductive age. Gynecol Endocrinol 2003;17: 125-31.

7. Kim CW, Shim HS, Jang H, Song YG. The effects of uterine artery embolization on ovarian reserve. Eur J Obstet Gynecol Reprod Biol 2016;206:172-6.

8. Siddle N, Sarrel P, Whitehead M. The effect of hysterectomy on the age at ovarian failure: identification of a subgroup of women with premature loss of ovarian function and literature review. Fertil Steril 1987;47:94-100.

9. Bukovsky I, Halperin R, Schneider D, Golan A, Hertzianu I, Herman A. Ovarian function following abdominal hysterectomy with and without unilateral oophorectomy. Eur J Obstet Gynecol Reprod Biol 1995;58:29-32.

10. Lee DY, Park HJ, Kim BG, Bae DS, Yoon BK, Choi D. Change in the ovarian environment after hysterectomy as assessed by ovarian arterial blood flow indices and serum anti-Mullerian hormone levels. Eur J Obstet Gynecol Reprod Biol 2010;151:82-5.

11. Barth MM, Spies JB. Ovarian artery embolization supplementing uterine embolization for leiomyomata. J Vasc Interv Radiol 2003; 14:1177-82.

12. Broekmans FJ, Kwee J, Hendriks DJ, Mol BW, Lambalk CB. A systematic review of tests predicting ovarian reserve and IVF outcome. Hum Reprod Update 2006;12:685-718.

13. Corson SL, Gutmann J, Batzer FR, Wallace H, Klein N, Soules MR. Inhibin-B as a test of ovarian reserve for infertile women. Hum Reprod 1999;14:2818-21.

14. Ellsworth $\mathrm{LR}$, Allen $\mathrm{HH}$, Nisker JA. Ovarian function after radical hysterectomy for stage IB carcinoma of cervix. Am J Obstet Gynecol 1983;145:185-8.

15. Muraji M, Sudo T, Iwasaki S, Ueno S, Wakahashi S, Yamaguchi S, et al. The effect of abdominal radical trachelectomy on ovarian reserve: serial changes in serum anti-Mullerian hormone levels. J Cancer 2012;3:191-5.

16. Buekers TE, Anderson B, Sorosky Jl, Buller RE. Ovarian function after surgical treatment for cervical cancer. Gynecol Oncol 2001;
80:85-8.

17. Ryu RK, Chrisman HB, Omary RA, Miljkovic S, Nemcek AA Jr, Saker $\mathrm{MB}$, et al. The vascular impact of uterine artery embolization: prospective sonographic assessment of ovarian arterial circulation. JVasc Interv Radiol 2001;12:1071-4.

18. Kim HS, Tsai J, Lee JM, Vang R, Griffith JG, Wallach EE. Effects of utero-ovarian anastomoses on basal follicle-stimulating hormone level change after uterine artery embolization with trisacryl gelatin microspheres. JVasc Interv Radiol 2006;17:965-71.

19. Kaump GR, Spies JB. The impact of uterine artery embolization on ovarian function. J Vasc Interv Radiol 2013;24:459-67.

20. Hu NN, Kaw D, McCullough MF, Nsouli-Maktabi H, Spies JB. Menopause and menopausal symptoms after ovarian artery embolization: a comparison with uterine artery embolization controls. JVasc Interv Radiol 2011;22:710-5.

21. Scheurig-Muenkler C, Poellinger A, Wagner M, Hamm B, Kroencke TJ. Ovarian artery embolization in patients with collateral supply to symptomatic uterine leiomyomata. Cardiovasc Intervent Radiol 2011;34:1199-207.

22. Tsikouras P, Manav B, Koukouli Z, Trypsiannis G, Galazios G, Souftas $D$, et al. Ovarian reserve after fibroid embolization in premenopausal women. Minim Invasive Ther Allied Technol 2017; 26:284-91

23. McLucas B, Voorhees WD 3rd, Snyder SA. Anti-Mullerian hormone levels before and after uterine artery embolization. Minim Invasive Ther Allied Technol 2018;27:186-90.

24. Tropeano G, Di Stasi C, Amoroso S, Gualano MR, Bonomo L, Scambia G. Long-term effects of uterine fibroid embolization on ovarian reserve: a prospective cohort study. Fertil Steril 2010;94: 2296-300.

25. McLucas B. Pregnancy following uterine artery embolization: an update. Minim Invasive Ther Allied Technol 2013;22:39-44.

26. Yokota A, Nakai A, Oya A, Koshino T, Araki T. Changes in uterine and ovarian arterial impedance during the periovulatory period in conception and nonconception cycles. J Obstet Gynaecol Res 2000;26:435-40

27. Chang WC, Huang SC, Sheu BC, Shih JC, Hsu WC, Chen SY, et al. Changes in uterine blood flow following laparoscopic myomectomy with or without uterine artery ligation on two- and threedimensional power Doppler ultrasound. Ultrasound Obstet Gynecol 2009;33:221-7.

28. Yildirim Y, Gultekin E, Kocyigit A, Yilmaz C, Ertopcu K, Arioz DT. Color Doppler analysis of pelvic arteries following bilateral internal iliac artery ligation for severe postpartum hemorrhage. Int J Gynaecol Obstet 2009;104:22-4.

29. Demirci F, Ozdemir I, Safak A, Ozden S, Somunkiran A. Comparison of colour Doppler indices of pelvic arteries in women with 
bilateral hypogastric artery ligation and controls. J Obstet Gynaecol 2005;25:273-4.

30. Kaplanoglu M, Karateke A, Un B, Gunsoy L, Baloglu A. Evaluation of uterine artery recanalization and doppler parameters after bilateral uterine artery ligation in women with postpartum hem- orrhage. Int J Clin Exp Med 2015;8:7823-9.

31. Greenwood LH, Glickman MG, Schwartz PE, Morse SS, Denny DF. Obstetric and nonmalignant gynecologic bleeding: treatment with angiographic embolization. Radiology 1987;164:155-9. 